\title{
Optimizing Drug Regimens in Cancer Chemotherapy
}

\author{
A. ILIADIS, D. BARBOLOSI \\ Department of Pharmacokinetics, Faculty of Pharmacy \\ University of Méditerranée \\ 27, bld. Jean Moulin, 13385 Marseilles Cedex 5 \\ FRANCE
}

\begin{abstract}
In cancer chemotherapy, it is important to design treatment strategies that ensure a desired rate of tumor cell kill without unacceptable toxicity. To optimize treatment, we used a mathematical model describing the pharmacokinetics of anticancer drugs, antitumor efficacy, and drug toxicity. This model was associated with constraints on the allowed drug concentrations and amounts, neutropenia, thrombopenia and their recovery levels before the next cycle of chemotherapy. Given a schedule of drug administrations, the mathematical model optimized the drug doses that can minimize the tumor burden while limiting hemotoxicity.
\end{abstract}

Key-Words: Dosage adjustment, Pharmacokinetics, Pharmacodynamics, Hemotoxicity

\section{Introduction}

Cancer chemotherapy is a systemic treatment, and thus it triggers toxic effects due to the interaction of the drug with normal cells, which prevents sufficient doses from being administered to obtain a complete cure. It becomes thus important to design treatment strategies for specific tumors and drug protocols for ensuring a desired rate of tumor cell kill without overdosing the host.

To design optimal chemotherapy strategies, one may use mathematical models describing the mechanisms of cytotoxic drug availability and action on tumor cell populations and expressing drug use constraints due to the subsequent toxicities. There are many approaches and models for reducing the tumor burden while keeping the side effects of the drugs at acceptable levels $[1,2]$. For most of these works, however, and it is difficult to correctly formulate and use the constraint related to drug-induced toxicity.

This study concerns the administration of two drugs and the optimization of regimens along several cycles of chemotherapy. The first part of this contribution presents the mathematical model, the constraints expressing toxicity induced by the treatment, and the criterion used to optimize drug administration. In the second part, to illustrate the optimization of regimens, we applied this general methodology to etoposide - cisplatin chemotherapy data, with comparisons between the optimized protocol and the traditional clinical protocol. Some prospects are discussed concerning drug resistance, and use of growth factors.

\section{Mathematical Modeling}

\subsection{Pharmacokinetics}

Compartment models are used to describe the kinetic behavior of the administered drugs:

$$
\dot{c}_{k}(t)=-f_{k} c_{k}(t)+\frac{u_{k}(t)}{V_{k}}
$$


with $c_{k}(0)=0$. In this model, the circulating concentrations, $c_{k}(t)$, for the two drugs, etoposide (VP16, $k=1$ ) and cisplatin (CDDP, $k=2$ ) are assumed to be efficient against the tumor proliferation. Parameters $V_{k}$ and $f_{k}$ are the volumes of distribution and the elimination rates associated to the compartments and $u_{k}(t)$ represent the input functions expressing the administration protocol for each drug $k$.

\subsection{Pharmacodynamics - efficacy}

Herein tumors were considered homogeneous, not only throughout their growth but also after being perturbed by an anticancer drug. Then, we assumed that this tumor grows according to a Gompertz - type growth equation [3] and that the cell loss term, due to the cytotoxicity, depends on the concentration of the drugs:

$$
\dot{n}(t)=\xi n(t) \ln \left[\frac{\theta}{n(t)}\right]-K\left(\bar{c}_{1}, \bar{c}_{2}\right) n(t)
$$

with $n(0)=n_{0}$, the initial tumor size. In this model, $n(t)$ denotes the number of tumor cells at time $t$, and $\theta$ and $\xi$ are the parameters of the Gompertz growth equation. The second term is the cell-loss term depending on the effective concentrations, $\bar{c}_{k} \triangleq$ $\left[c_{k}(t)-C_{\min }\right] H\left[c_{k}(t)-C_{\min }\right]$, where $C_{\min }$ is a threshold below which no tumor cells are killed [3]. In the loss term, $H($.$) is the Heaviside step$ function and $K\left(\bar{c}_{1}, \bar{c}_{2}\right)$ is the function expressing the constant-cell-kill hypothesis, expected to be valid when the cells are sensitive to the anticancer drugs. Herein, the choice was $K\left(\bar{c}_{1}, \bar{c}_{2}\right)=$ $\kappa_{1} \bar{c}_{1}+\kappa_{2} \bar{c}_{2}+\kappa_{12} \bar{c}_{1} \bar{c}_{2}$, the last term expressing the potentialization in drug cytotoxicity induced by the presence of two drugs. Other mathematical models could be formulated and tested against data from experimental and clinical tumors.

\subsection{Pharmacodynamics - toxicity}

When an anticancer drug is introduced into the body, both normal and malignant cells are killed. In hemopoietic system, the release of marrow cells to proliferation, maturation, and to the blood is greatly disturbed by chemotherapy.
Absolute neutrophil count (ANC) and platelet count (PLC) fall to low levels, and much care must be taken to maintain the status of the patient. Because of the onset of toxic effects on ANC and PLC, a more extensive formulation must include the normal cell kinetics that will be affected by the drug. Appropriate constraints must be included to ensure that the decay of hemopoietic system populations does not become excessively large.

The dynamical toxicity of chemotherapy on $\mathrm{ANC}$ and PLC can be described by:

$$
\dot{w}_{s}(t)=R_{s}\left(\widetilde{c}_{1}, \widetilde{c}_{2}\right)-\left(\nu_{s}+\mu_{s 1} \widetilde{c}_{1}+\mu_{s 2} \widetilde{c}_{2}\right) w_{s}(t)
$$

with $w_{s}(0)=w_{s 0}$. In this model, $w_{s}(t)$ denotes the hematologic cell counts $(s=1$ for ANC and $s=2$ for PLC). Because myelosuppression occurs in the bone marrow site, the drug toxicity is expressed by delayed concentration levels, $\widetilde{c}_{k} \triangleq c_{k}\left(t-\tau_{s}\right)$ [4]. The loss term introduces the drug toxicity on the $w_{s}(t)$ circulating cells by $\mu_{s 1} \widetilde{c}_{1}$ and $\mu_{s 2} \widetilde{c}_{2}$ and it accounts for the physiological losses by $\nu_{s}$. The first term $R_{s}\left(\widetilde{c}_{1}, \widetilde{c}_{2}\right)$ expresses the production rate of hematologic cells; it is assumed to vary between $\nu_{s} w_{s 0}$ (the normal production rate) and $\nu_{s} w_{s 0} / 100$ according to a double exponential model that involves the delayed drug levels $\lambda_{s 1} \widetilde{c}_{1}$ and $\lambda_{s 2} \widetilde{c}_{2}$.

\section{Constraints}

The anticancer drug should be scheduled to ensure that the patients tolerate the toxic side effects. Then, pharmacokinetic (PK) constraints are formulated to specify the maximum allowed drug amounts and circulating concentrations, and hematologic prerequisites constrain the time-courses of ANC and PLC.

\subsection{Pharmacokinetic constraints}

A local limit, $C_{\max , k}$, is put on $c_{k}(t)$ because there must be an upper bound for the drug concentration:

$$
c_{k}\left(t_{i}\right) \leq C_{\max , k}
$$

where $t_{i}$ are the times of maximum concentrations depending on the administration protocol. 
A global control may be $D_{\max , k}$, the maximum allowed drug amount per cycle of chemotherapy:

$$
\sum_{i=1}^{N} d_{i k} \leq D_{\max , k}
$$

where $d_{i k}$ is the amount of the $k$-th drug delivered by the $i$-th administration.

\subsection{Hematologic constraints}

First, we formulate simply that hematologic cell counts $w_{s}(t)$ must remain controlled at levels higher than a fixed down level, $W_{A s}$. This constraint ensures absolute protection from neutropenia and thrombopenia. It is transformed into a discrete form:

$$
w_{s}\left(t_{j}\right) \geq W_{A s} \quad t_{j}=\tau+j \Delta t
$$

with $j=0, \ldots,\left[\frac{T}{\Delta t}\right] . T$ is the cycle interval and $[z]$ denotes the integer part of the $z$. The sampling interval $\Delta t$ is chosen to be at least twice as high as the highest frequency determined by power spectra analysis of the sampled signal generated by equation 3 . This rule guarantees that no information is lost when a continuous signal is to be correctly presented in a discrete form. This analysis indicates that the sampling interval must be less than $0.5 \min _{s, k}\left[\left(\nu_{s}+\mu_{s k} C_{\max , k}\right)^{-1}\right]$.

A second constraint was introduced to prevent the hematologic cell counts from staying too long below a fixed upper level, $W_{R k}$. Let $t_{R s}(t)$ be the time over which $w_{s}(t)$ remains below $W_{R s}$; this time may be evaluated from the following differential equation:

$$
\dot{t}_{R s}(t)=H\left[W_{R s}-w_{s}(t)\right]
$$

with $t_{R s}(0)=0$. We constrain the time $t_{R s}(T)$ to be less than $T_{R s}$ :

$$
t_{R s}(T) \leq T_{R s}
$$

This constraint expresses a relative protection from neutropenia and thrombopenia.

A third constraint was used to ensure a minimum recovery of the hematologic cell counts at the end of the present cycle and before the beginning of the next one:

$$
w_{s}(T) \geq W_{C s}
$$

\section{Optimal Drug Regimens}

An optimal control problem may be formulated as follows: Determine the positive continuous time optimal input function that minimizes the tumor size subjected to the state equations 1 to 3 and 7 , while satisfying the constraints 4 to 6,8 and 9 [1]. Nevertheless, it is not always easy to obtain the theoretical optimal input function because drug administration is constrained by the route of administration, intra- or extra-vascular. One solution is to minimize an objective function with respect to a chosen class of input functions.

Let this class of input functions, corresponding to intermittent or continuous intravenous infusions covering the entire cycle interval $T$, be expressed by $N$ piecewise constant functions defining the administration protocol as follows:

$$
u_{k}(t)= \begin{cases}d_{1 k} / t_{1} & 0 \leq t<t_{1} \\ d_{i k} /\left(t_{i}-t_{i-1}\right) & t_{i-1} \leq t<t_{i}\end{cases}
$$

with $i=2, N$. In this model, $d_{i k}$ are the amounts infused between the start- and the endof-infusion times $t_{i-1}$ and $t_{i}$, respectively $\left(d_{i k} \equiv 0\right.$ when no administration). The total cycle interval is $t_{N} \equiv T$, and $t_{i} \quad i=1, N$ are the times of the maximum concentrations in constraint 4 . For compactness, let $\underline{d}$ be the vector of $d_{i 1}$ and $d_{i 2}$. We propose setting the schedule of drug administrations at the $N$ preassigned switching times $t_{i}$ and then computing in equation 10 the optimal amounts $\underline{d}^{*}$.

The above remarks enable the optimization problem to be transformed into a more clinically tractable problem that can be formulated as follows: After setting the schedule of drug administrations $t_{i} \quad i=1, N$, the tumor time-course $n(t)$ may be obtained for a given $\underline{d}$; let $n\left(t^{*}\right)$ be the minimum value of tumor burden at time $t^{*}$; among all possible values of $\underline{d}$, we seek $\underline{d}^{*}$ that leads to the lowest $n\left(t^{*}\right)$ :

$$
\left[\begin{array}{ll}
\underline{d}^{*} & t^{*}
\end{array}\right]=\arg \min [n(t)]
$$


subject to the state equations 1 to 3 and 7 , and satisfying constraints 4 to 6,8 and 9 .

In these circumstances, the initial constrained optimal control problem is converted into a nonlinear optimization parameter problem. Although such an approach yields only a sub-optimal strategy for the given system, the resulting trade-off with complexity is of considerable attractiveness to determine efficient protocols for cancer chemotherapy. This procedure was applied by others in cancer chemotherapy $[5,6]$, and it is commonly used in clinical PKs for optimal dosage regimen computation $[7,8]$.

Nonlinear programming and numerical integration techniques with MATLAB [9] were used to solve this problem.

\section{Simulation Study}

This study provides a general example for treatment of solid tumors by an association of anticancer drugs. Usually, the cycle of drugs is repeated at about three-week intervals for the traditional clinical protocol with VP16 (30 - min infusion of $0.63 \mathrm{~g}$ at days 1, 2 and 3 ) and CDDP ( $1-\mathrm{h}$ infusion of $0.175 \mathrm{~g}$ at day 1$)$. In this study, we propose to design a new, densified protocol on a 14-day cycle interval $(T=14 \mathrm{~d})$ and to evaluate over 3 cycles of chemotherapy the performances of the traditional protocol and that of the optimized protocol. For the latter, we propose to apply daily constant-rate regular infusions at evenly spaced intervals over the cycle interval so that infusion rates may change each day. In these conditions, the optimization problem 11 is a nonlinear programming problem with 29 parameters.

\subsection{The data}

The model parameter values were set according to the published data [10]. The PK parameters were set at $V_{1}=25$ and $V_{2}=401, f_{1}=2$ and $f_{2}=0.1 \mathrm{~d}^{-1}$.

For the efficacy model in pharmacodynamics (PD), $n_{0}$ was set at $30 \mathrm{~g}, \theta=1 \mathrm{~kg}, \xi=0.006$ $\mathrm{d}^{-1}$ (without treatment a tumor doubles in size in $40 \mathrm{~d}$ ). Also, $C_{\min }=10^{-4} \mathrm{~g} \cdot \mathrm{l}^{-1}, \kappa_{1}=10$, $\kappa_{2}=5 \mathrm{~d}^{-1} \cdot \mathrm{g}^{-1} \cdot \mathrm{l}$, and $\kappa_{12}=2 \cdot 10^{4} \mathrm{~d}^{-1} \cdot \mathrm{g}^{-2} \cdot \mathrm{l}^{2}$.

For the toxicity model in PDs, data for ANC were $\nu_{1}=1 \mathrm{~d}^{-1}, \lambda_{11}=450, \lambda_{12}=900$, and $\mu_{11}=40 \mathrm{~d}^{-1} \cdot \mathrm{g}^{-1} \cdot \mathrm{l}, w_{10}=4 \cdot 10^{6} \mathrm{ml}^{-1}$, and $\tau_{1}=5 \mathrm{~d}$. The PLC data were $\nu_{2}=0.3$ $\mathrm{d}^{-1}, \lambda_{21}=0, \lambda_{22}=1500$, and $\mu_{21}=40$ $\mathrm{d}^{-1} \cdot \mathrm{g}^{-1} \cdot \mathrm{l}, w_{20}=300 \cdot 10^{6} \mathrm{ml}^{-1}$, and $\tau_{2}=1 \mathrm{~d}$. For both ANC and PLC, CDDP is assumed to have no toxicity on circulating hematologic cells, $\mu_{12}=\mu_{22}=0$.

For the PK constraints, we set $C_{\max , 1}=10$ and $C_{\max , 2}=5 \mathrm{mg} \cdot \mathrm{l}^{-1}, D_{\max , 1}=1$, and $D_{\max , 2}=0.18 \mathrm{~g}$. The ANC hematologic constraints were $W_{A 1}=0.5, W_{R 1}=1, W_{C 1}=1.5$, and the PLC constraints, $W_{A 2}=20, W_{R 2}=$ $50, W_{C 2}=10010^{6} \mathrm{ml}^{-1}$. The maximum allowed times for neutropenia and thrombopenia were set at $T_{R 1}=T_{R 2}=4 \mathrm{~d}$ per cycle and the sampling interval was $0.35 \mathrm{~d}$.

\section{$5.2 \quad$ Results}

First, we simulated the fate of drug concentrations, tumor size, and ANC and PLC counts through the traditional clinical protocol. Over the 3 cycles of treatment, we used total amounts of 1.89 and $0.525 \mathrm{~g}$ for VP16 and CDDP respectively. This treatment led to a minimal tumor mass of $5.7 \mathrm{~g}\left(18.8 \%\right.$ reduction of $\left.n_{0}\right)$ at $32 \mathrm{nd}$ day. Nevertheless, the traditional protocol was followed by severe absolute neutropenia at cycles 2 and 3, and relative neutropenia and thrombopenia of 15.1 and 22.8 d respectively, cumulated on the 3 cycles. These findings on tumor fate as well as on the simulated ANC and PLC count characteristics are consistent with the literature $[10,11]$.

The Figure shows optimized drug rates $u^{*}(t)$, and the dynamics of tumor size and of the ANC and PLC counts. Over the 3 cycles of treatment, we used total amounts of 2.64 and 0.36 $\mathrm{g}$ for VP16 and CDDP respectively. The form of $u^{*}(t)$ turns out to be per cycle a combination of high doses at the start of cycle and then continuous infusions of moderate doses. CDDP is designed to be given only at the first day of each cycle. Note that in the first day, the opti- 
mal drug dose induces maximum plasmatic levels, so that the ANC and PLC counts are driven down to their lower levels $W_{A s}$. Then, the drug infusion rates are chosen to maintain these levels just for a time $(3-4$ d) compatible with the relative constraint 8 . After that time and because of the constraint 5, the VP16 administration is stopped and the patient rests for a $10-\mathrm{d}$ interval before starting the next cycle of chemotherapy. Initially, for the $4-5 \mathrm{~d}$ of drug administrations at each cycle, the number
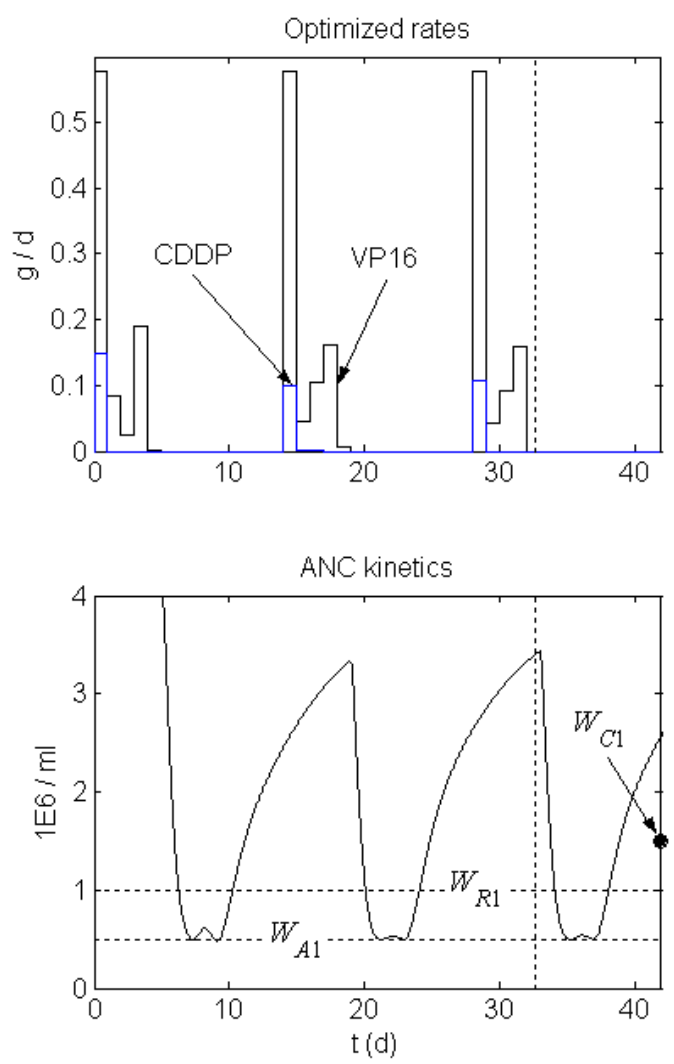

\section{Discussion and Prospects}

The experimental form of the optimal drug administration confirms the theoretical findings of Murray [12]. Indeed, in a context with general cell-loss functions on normal and tumor cells, constrained optimal control theory defines the optimal drug administration to be high dose chemotherapy up to saturation of constraints attached to normal cell toxicity and then moderate drug administration to manage active con- of tumor cells decreases rapidly and then, when chemotherapy is stopped, the tumor grows again slowly. Note that its minimum value $(7.1 \mathrm{~g}$, reduction of $23.5 \%$ ) is achieved at the $32 \mathrm{nd}$ day.

In conclusion, the optimized protocol is slightly less efficient that the traditional one, but it is exempt from any toxicity. The optimal protocol has the advantages and avoids the drawbacks of the traditional clinical protocols because it perfectly monitors toxicities while reducing the tumor mass.
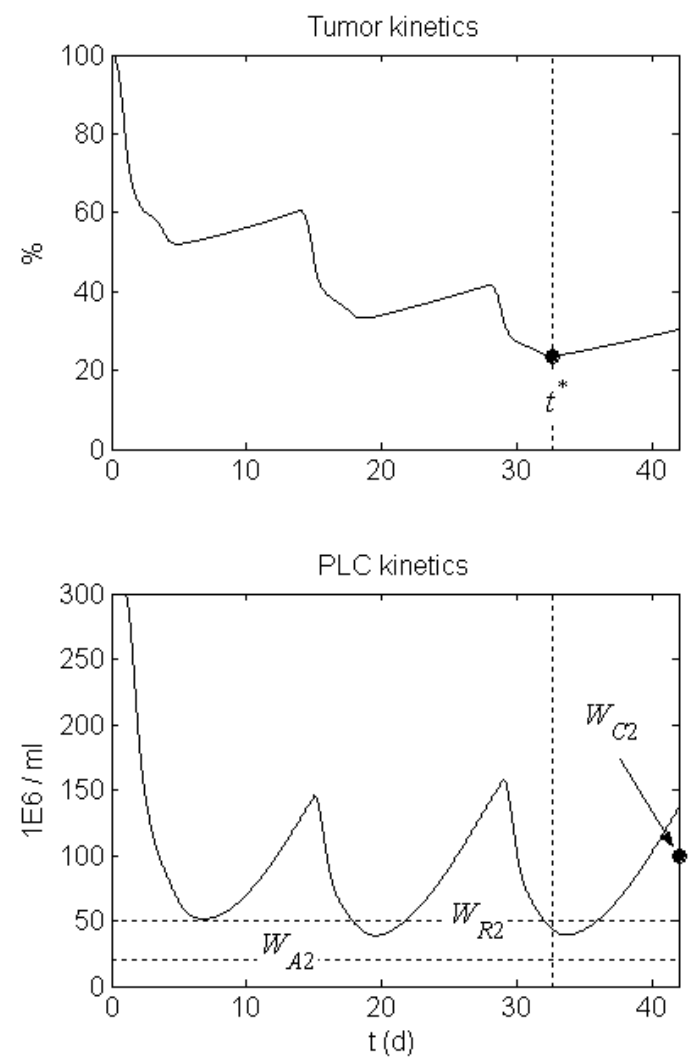

straints. The problem is fully constrained and the form of the optimal inputs is completely dictated by the extent of constraints associated to the PD - toxicity model.

The constant-cell-kill hypothesis, valid for sensitive tumor cells, does not appear valid for clinical tumors, for which increasing drug resistance can be expected to decrease the cellkill factor. Chemotherapy is not effective in many instances because tumor resistance devel- 
ops. There is an obvious need to incorporate the effects of drug resistance into tumor growth models in order to evaluate the response of the tumor to chemotherapy [13].

Hemopoietic growth factors (G-CSF) used in conjunction with cancer chemotherapy have led to safer delivery of standard-dose chemotherapy regimens [14]. Nevertheless, it is not known how G-CSF can be administered to improve the hematologic status during chemotherapy. Using a mathematical model for G-CSF dynamics, it is conceivable to optimize their rate of administration and then alter the profile of dose-limiting toxicity, enabling investigators to apply larger doses of anticancer drugs to be more effective [15].

Although many anticancer drugs have been in clinical use for over 20 years, their optimum schedule of administration is still not clear. Mathematical models need to be developed to better understand how to implement this work and perhaps to elaborate new optimal treatment strategies. Drug resistance modeling and optimization of G-CSF administration should be further investigated. We hope that these findings will be the beginning of considerations for definitively discarding static aspects in modern PK - PD modeling.

\section{References:}

[1] GW. Swan, Applications of optimal control theory in biomedicine, Marcel Dekker, 1984

[2] GW. Swan, Role of optimal control theory in cancer chemotherapy, Mathematical Biosciences, Vol.101, 1990, pp.237-284

[3] WP. Peters, R. Dansey, New concepts in the treatment of breast cancer using high-dose chemotherapy, Cancer Chemotherapy Pharmacology, Vol.40, 1997, pp.88-93

[4] AS. Fokas, JB. Keller, BD. Clarkson, Mathematical model of granulocytopoiesis and chronic myelogenous leukemia, Cancer Research, Vol.51, 1991, pp.2084-2091

[5] RB. Martin, Optimal control drug scheduling of cancer chemotherapy, Automatica, Vol.28, 1992, pp.1113-1123
[6] MK. Sundareshan, RS. Fundakowski, Periodic optimization of a class of bilinear systems with application to control of cell proliferation and cancer therapy, IEEE Transactions on Systems, Man, and Cybernetics, Vol.15, 1985, pp.102-115

[7] A. Iliadis, R. Bruno, JP. Cano, Dynamical dosage regimen calculations in linear pharmacokinetics, Computers and Biomedical Research, Vol.21, 1988, pp.203-220

[8] A. Iliadis, D. Barbolosi, Optimizing drug regimens in cancer chemotherapy by an efficacy - toxicity mathematical model, Computers and Biomedical Research, Vol.33, 2000, pp.211-226

[9] MATLAB. High-performance numeric computation and visualization software, Release 6.5, The Math Works, 2002

[10] DC. Ihde, JL. Mulshine, BS. Kramer, SM. Steinberg, RI. Linnoila et al, Prospective randomized comparison of high-dose and standard-dose etoposide and cisplatin chemotherapy in patients with extensive-stage small-cell lung cancer, Journal of Clinical Oncology, Vol.12, 1994, pp.2022-2034

[11] PI. Clark, ML. Slevin, SP. Joel, RJ. Osborne, DI. Talbot et al, A randomized trial of two etoposide schedules in small-lung cancer: The influence of pharmacokinetics on efficacy and toxicity, Journal of Clinical Oncology, Vol.12, 1994, pp.1427-1435

[12] JM. Murray, Optimal control for a cancer chemotherapy problem with general growth and loss functions, Mathematical Biosciences, Vol.98, 1990, pp.273-287

[13] JC. Panetta, A mathematical model of drug resistance: heterogeneous tumors, Mathematical Biosciences, Vol.147, 1998, pp.41-61

[14] N. Hayashi, H. Kinoshita, E. Yukawa, S. Higuchi, Pharmacokinetic and pharmacodynamic analysis of subcutaneous recombinant human granulocyte colony stimulating factor (lenograstim) administration, Journal of Clinical Pharmacology, Vol.39, 1999, pp.583-592

[15] N. Saijo, Chemotherapy: The more the better? Overview, Cancer Chemotherapy Pharmacology, Vol.40, 1997, pp.100-106 